\title{
Galactic archaeology for amateur astronomers: RR Lyrae stars as tracers of the Milky Way formation
}

\author{
Julio A. Carballo-Bello, David Martínez-Delgado \& Jürgen Fliri \\ Instituto de Astrofísica de Canarias \\ Vía Láctea s/n, 38205 La Laguna, Tenerife, Spain \\ email: jacb@iac.es
}

\begin{abstract}
Cosmological models predict that large galaxies like the Milky Way formed from the accretion of smaller stellar systems. The most spectacular of these merger events are stellar tidal streams, rivers of stars and dark matter that envelop the discs of spiral galaxies. We present a research project for a collaboration with amateur astronomers in the study of the formation process of our Galaxy. The main objective is the search for RR Lyrae variable stars in the known stellar streams (Sagitarius, Monoceros, Orphan, etc) a project that can be carried out using small telescopes. The catalogue of candidate variable stars were selected from SDSS data based in colour criteria and it will be sent to interested amateur astronomers who wish to participate in scientific research in one of the most active and competitive topics in Galactic astronomy.
\end{abstract}

Keywords. RR Lyrae stars, Galaxy, galaxy formation, Galactic halo, amateur astronomers

\section{Introduction}

RR Lyrae are variable stars with radial pulsations with a period varying from 0.2 to 1.2 days and present a magnitude change with amplitudes in the range $0.2-2$. The instability strip in the colour-magnitude diagram lies between 0.18 and 0.40 in $B-V$ colour $(7,400$ and $6,100 \mathrm{~K}$ respectively). The principal advantage of RR Lyrae is that they have aproximately the same intrinsic brightness, $\left\langle M_{V}\right\rangle=+0.7 \pm 0.2$, becoming an useful tool in the measurement of distances up to $100 \mathrm{kpc}$. This type of star is present in old stellar populations such as globular clusters, the bulge and halo of the Milky Way and are at least $12 \mathrm{Ga}$ old.

\subsection{Hierarchical formation and the RR Lyrae population}

Within the hierarchical framework for galaxy formation, galaxies are expected to form and evolve through mass infall and through the successive coalescence of smaller, distinct building blocks, such as satellite galaxies merging with their parent galaxy. This framework predicts distinct tidal stellar streams in and around large galaxies and at $d \geqslant 10$ kpc such streams should remain detectable as coherent stellar over-densities for billions of years. Over the last years, different large scale CCD surveys (Sloan Digital Sky Survey, 2MASS, QUEST) have probed for the first time the presence of a significant amounts of these substructures in the halo of the Milky Way (MW) in form of long tidal streams or stellar clumps (Newberg et al. 2002; Majewski et al. 2003; Martínez-Delgado et al. 2001) which have been interpreted as the fossil records of the hierarchical Galaxy formation. On the other hand, N-body simulations of these merger events (Martínez-Delgado 
et al. 2004; Peñarrubia et al. 2005; Johnston et al. 2005) have also been extensively used to associate detected tidal debris with their dwarf progenitor galaxies with the aim of constraining the dynamical history of the progenitor satellites and the properties of the MW's dark matter halo. This observational and theoretical effort has led to an comprensive understanding of the two largest tidal streams discovered so far in our Galaxy: the tidal stream of the Sagittarius dwarf galaxy, which wraps the MW in an almost polar orbit (Majewski et al. 2003; Martínez-Delgado et al. 2001) and the Monoceros tidal stream (Yanny et al. 2003; Ibata et al. 2003) a low-latitude tidal stream whose progenitor moves on a prograde, nearly circular orbit.

In addition to these two large tidal streams, these large-scale surveys have revealed the presence of numerous stellar over-densities possibly associated with unknown merger events in the halo. This project is devoted to confirm whether these observed substructures could be related with the fossil records of the hierarchical formation of the Milky Way and to complete in this way the number of merger events in our Galaxy, which can be directly compared with the predictions of the Cold Dark Matter cosmological models (Bullock \& Johnston 2005).

RR Lyrae are good tracers of this phenomenon since they are at least 12 Ga old and are present in old stellar populations such as the tidal streams. The discovery of RR Lyrae stars in the Galactic halo with distances compatible with the known streams would prove that these structures are not part of the Milky Way but they are the result of ancient merger processes. The very well-known period-luminosity relation for these stars would allow us to measure distances to the streams improving our models designed to represent these systems.

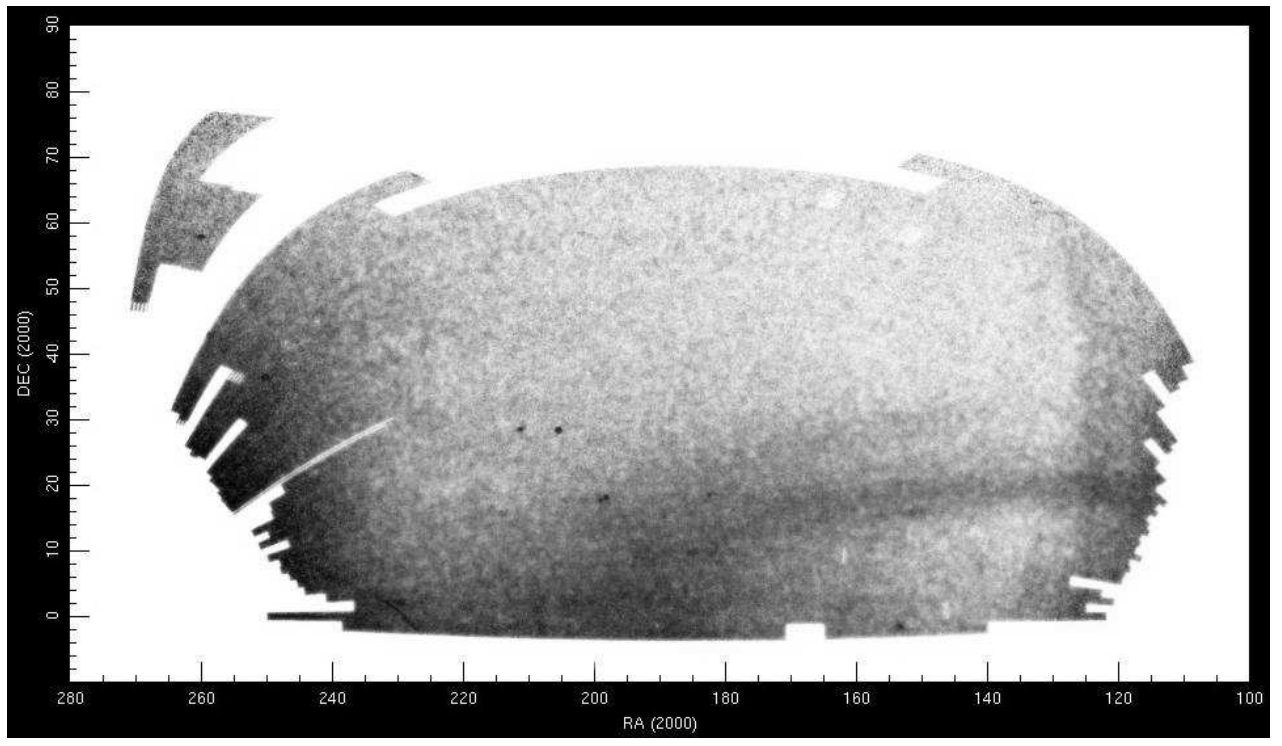

Figure 1. Field of streams: density map created from SDDS data of the region covered by this survey. There are different known substructures in this area (Sagittarius, Orphan and Monoceros stream) where we plan to detect RR Lyrae stars. With this purpose, we will concentrate only in the regions where the streams cross the SDSS sky. 


\section{Collaboration with amateur astronomers}

Following the criteria found by Ivezić et al. (2005), we have elaborated a list of candidates RR Lyrae stars from the Sloan Digital Sky Survey (a quarter of the visible sky from the northern hemisphere). We have focused in the three known streams clearly visible in SDSS data - Sagittarius, Monoceros and Orphan streams - to confirme their tidal origin. This project can be carried out using small telescopes since they have been converted in a powerful tool in recent years to study variable objects and amateur astronomers have much experience in this topic. This combination has inspired us to develop an observational program whereby all astronomers are invited to participate in this key research field.

\section{References}

Bullock, J.S. \& Johnston, K.V. 2005, ApJ, 635, 931

Ibata, R.A., et al. 2003, MNRAS, 340, L21

Ivezić, Ž., Vivas, A.K., Lupton, R.H. \& Zinn, R. 2005, AJ, 129, 1096

Johnston, K.V., Law, D.R., Majewski, S.R. 2005, ApJ, 619, 800

Majewski, S.R., Strutskie, M.F., Weinberg, M.D. \& Ostheimer, J.C. 2003, ApJ, 599, 1082

Martínez-Delgado, D., Aparicio, A., Gómez-Flechoso, M. A. \& Carrera, R. 2001, ApJ, 549, L199

Martínez-Delgado, D., Gómez-Flechoso, M.A., Aparicio, A. \& Carrera, R. 2004, ApJ, 601, 242

Martínez-Delgado, D. et al. 2008, ApJ, 689, 184

Newberg, H. J., et al. 2002, ApJ, 569, 245

Peñarrubia, J., et al. 2005, $A p J, 626,128$

Yanny, B., et al. 2003, $A p J, 588,824$ 